Research Article

\title{
On the Stability of Fractional Differential Equations Involving Generalized Caputo Fractional Derivative
}

\author{
Minh Duc Tran, ${ }^{1,2}$ Vu Ho, ${ }^{3}$ and Hoa Ngo Van $\mathbb{1 D}^{2,4}$ \\ ${ }^{1}$ Faculty of Electrical Electronics Engineering, Ton Duc Thang University, Ho Chi Minh City, Vietnam \\ ${ }^{2}$ Division of Computational Mathematics and Engineering, Institute for Computational Science, Ton Duc Thang University, \\ Ho Chi Minh City, Vietnam \\ ${ }^{3}$ Faculty of Mathematical Economics, Banking University of Ho Chi Minh City, Vietnam \\ ${ }^{4}$ Faculty of Mathematics and Statistics, Ton Duc Thang University, Ho Chi Minh City, Vietnam
}

Correspondence should be addressed to Hoa Ngo Van; ngovanhoa@tdtu.edu.vn

Received 28 September 2019; Accepted 16 January 2020; Published 15 February 2020

Academic Editor: Gisele Mophou

Copyright (c) 2020 Minh Duc Tran et al. This is an open access article distributed under the Creative Commons Attribution License, which permits unrestricted use, distribution, and reproduction in any medium, provided the original work is properly cited.

This work presents the results of the global existence for fractional differential equations involving generalized Caputo derivative with the case of the fractional order derivative $\alpha \in(1,2)$. In addition, the Ulam-Hyers-Mittag-Leffler stability of the given problems is also established.

\section{Introduction}

In recent years, there are a vast number of various concepts for fractional integrals and derivatives, such as Riemann-Liouville, Riesz, Grünwald-Letnikov, Hadamard, and Caputo derivatives and/or integrals. One can notice that most of the research results on the topics of fractional differential equations involving Riemann-Liouville and Hadamard fractional derivatives have been paid more and more attention by a large number of mathematicians because of the interesting and their applications. For more details on fractional calculus theory and interesting applications, one can see the monographs and the interesting papers in [1-6] and the references cited therein. However, both of the definitions of Hadamard and Riemann-Liouville fractional derivatives have their own disadvantages as well; one of which is that the derivative of a constant is not equal to zero. Then, to overcome the disadvantage of two types of these fractional derivatives, the Caputo and Caputo-Hadamard fractional derivatives were proposed. In the past decade, in $[7,8]$, Katagampola has proposed a new generalized concept of the fractional derivative, the so-called Caputo-Katugampola, that unifies the definitions of Caputo and Caputo-Hadamard fractional derivatives into a single form. The parameter family $\rho$ of Caputo-Katugampola fractional derivative, ${ }^{C} D_{a^{+}}^{\alpha, \rho}$, of the noninteger order $\alpha$ allows one to interpolate two types of the Caputo and Caputo-Hadamard fractional derivatives. Other approaches of fractional operators based on using very general kernel functions have been also proposed in $[1,9]$. These approaches relate to the various real data corresponding to different complex systems requiring different kernel functions. For more details, on Caputo-Katugampola fractional derivative and interesting applications, one can see the papers [1, 2, 10-15] and the references cited therein. Very recently, the motivation behind the approach of Caputo-Katugampola fractional operator relates to the chaos problems in fractional dynamical systems suggested in the security of image encryption $[16,17]$ and in quantum mechanics [12].

During the past two decades, a large number of mathematicians have paid great attention to the studies of the concepts of Ulam's stability because of its usefulness in many applications such as numerical analysis and optimization, where finding the exact solutions is quite difficult. In fact, it is not easy to get exact solutions to most of the problems of 
fractional differential equations. Therefore, it is vital to develop the concepts of Ulam's stability for these problems because we need not obtain the exact solutions of the given problems when we study the properties of Ulam's stability. This theory helps us getting an efficient and reliable technique for approximately solving fractional differential equations because there exists a close exact solution when the given problem is Ulam stable. More details from historical point of view and recent developments of such stabilities are reported in $[9,17-30]$ and the references cited therein. So, the motivation for the elaboration of this paper is the investigation of some kinds of the Ulam-Hyers stability for the following problem involving the concept of Caputo-Katugampola fractional derivative with the case of the $\alpha \in(1,2)$ :

$$
\begin{aligned}
{ }^{C} D_{a^{+}}^{\alpha, \rho} \psi(t) & =f(t, \psi(t)), \\
\psi(a) & =\psi_{1}, \\
\psi^{\prime}(a) & =\psi_{2}, \\
t & \in[a, b],
\end{aligned}
$$

where $\alpha \in(1,2), \rho>0$ is a real parameter, $f:[a, b] \times \mathbb{R}^{d} \longrightarrow \mathbb{R}^{d}$ is a nonlinear continuous function, and ${ }^{C} D_{a^{+}}^{\alpha, \rho}$ is the Caputo-Katugampola fractional derivative.
Based on (1), the parameter $\rho$ allows one to get the initial value problem involving the Caputo fractional derivative if $\rho$ tends to 1 , and the initial value problem with the concept of Caputo-Hadamard fractional derivative if $\rho$ tends to $0^{+}$. Our aim in this paper is to discuss the global existence of solutions of problem (1) by using Schauder's and Weissinger's fixed point theorem. In addition, some kinds of the Ulam-Hyers stability of problem (1) are also established. The rest of this paper is arranged as follows: some fundamental theories of Caputo-Katugampola fractional calculus are introduced in Section 2. Section 3 is devoted to discuss the global existence of solutions of problem (1), and the stability of problem (1) is presented in Section 4.

\section{Fundamental Theorems of Fractional Analysis}

In this section, some definitions and basic results will be briefly presented which will be used throughout the paper. Let $C\left([a, b], \mathrm{R}^{d}\right)$ be the space of vector-valued continuous functions $\psi$ from $[a, b] \longrightarrow \mathrm{R}^{d}$ endowed with the norm $\psi_{0}=$ $\sup _{t \in[a . b]}\|\psi(t)\|$, where $\|\cdot\|$ is the vector norm in the $d$-dimensional Euclidean space. Denote by $C_{\gamma}\left([a, b], \mathbb{R}^{d}\right)$ the weighted space of continuous functions given by

$$
C_{\gamma}\left([a, b], \mathbb{R}^{d}\right)=\left\{\psi:(a, b] \longrightarrow \mathbb{R}^{d}:\left(\frac{t^{\rho}-a^{\rho}}{\rho}\right)^{\gamma} \psi(t) \in C\left([a, b], \mathbb{R}^{d}\right)\right\}
$$

where $\gamma \in(0,1]$.

Let $\alpha>0$, then the Riemann-Liouville generalized fractional integral of $\psi$ is defined by (see [7])

$$
\psi_{\alpha, \rho}(t):=\left(I_{a^{+}}^{\alpha, \rho} \psi\right)(t)=\frac{\rho^{1-\alpha}}{\Gamma(\alpha)} \int_{a}^{t} s^{\rho-1}\left(t^{\rho}-s^{\rho}\right)^{\alpha-1} \psi(s) \mathrm{d} s .
$$

Let $\alpha>0$, then the Riemann-Liouville generalized derivative of $\psi$ is defined by (see [7])

$$
\left({ }^{R L} D_{a^{+}}^{\alpha, \rho} \psi\right)(t)=\left(t^{1-p} \frac{\mathrm{d}}{\mathrm{d} t}\right)^{n} I_{a^{+}}^{n-\alpha, \rho} \psi(t)=\frac{\rho^{\alpha-n+1}}{\Gamma(n-\alpha)}\left(t^{1-p} \frac{\mathrm{d}}{\mathrm{d} t}\right)^{n} \int_{a}^{t} s^{\rho-1}\left(t^{\rho}-s^{\rho}\right)^{n-\alpha-1} \psi(s) \mathrm{d} s, \quad t>a,
$$

where $n=\alpha$.

Let $\alpha>0$, then the Caputo-generalized fractional derivative of $\psi$ denoted by $\left({ }^{C} D_{a^{+}}^{\alpha, \rho} \psi\right)$ is defined by

$$
\left({ }^{C} D_{a^{+}}^{\alpha, \rho} \psi\right)(t)={ }^{R L} D_{a^{+}}^{\alpha, \rho}\left[\psi(t)-\sum_{k=0}^{n-1} \frac{\left(t^{\rho}-a^{\rho}\right)^{k}}{\rho^{k} k !}\left[\left(t^{1-p} \frac{\mathrm{d}}{\mathrm{d} t}\right)^{k} \psi(t)\right]_{t=a}\right] .
$$

By putting $\psi^{(p, k)}(t):=\left(t^{1-p}(\mathrm{~d} / \mathrm{d} t)\right)^{k} \psi(t)$, we have that

$$
\begin{aligned}
\left({ }^{C} D_{a^{+}}^{\alpha, \rho} \psi(t)\right. & ={ }^{R L} D_{a^{+}}^{\alpha, \rho} \psi(t)-\sum_{k=0}^{n-1} \frac{\psi^{(p, k)}(a)}{\rho^{k} k !}{ }^{R L} D_{a^{+}}^{\alpha, \rho}\left(t^{\rho}-a^{\rho}\right)^{k} \\
& ={ }^{R L} D_{a^{+}}^{\alpha, \rho} \psi(t)-\sum_{k=0}^{n-1} \frac{\psi^{(p, k)}(a)}{\Gamma(k-\alpha+1)}\left(\frac{t^{\rho}-a^{\rho}}{\rho}\right)^{k-\alpha},
\end{aligned}
$$

where $\quad \psi^{(\rho, k)}(a):=\left[\left(t^{1-p}(\mathrm{~d} / \mathrm{d} t)\right)^{k} \psi(t)\right]_{t=a} . \quad$ If $\quad \psi \in C^{n}$ $\left([a, b], \mathbb{R}^{d}\right)$, then we have that (see [7]) 


$$
\left({ }^{C} D_{a^{+}}^{\alpha, \rho} \psi\right)(t)=\frac{\rho^{\alpha-n+1}}{\Gamma(n-\alpha)} \int_{a}^{t} s^{(\rho-1)(1-n)}\left(t^{\rho}-s^{\rho}\right)^{n-\alpha-1} \frac{\mathrm{d}^{n} \psi}{\mathrm{d} s^{n}}(s) \mathrm{d} s, \quad t \geq a .
$$

We observe that

$$
\begin{aligned}
& \left\|\psi_{\alpha, \rho}\right\|_{0}:=\sup _{t \in[a, b]}\left\|\psi_{\alpha, \rho}(t)\right\| \leq\|\psi\|_{0} \frac{\rho^{1-\alpha}}{\Gamma(\alpha)} \int_{a}^{t} s^{\rho-1}\left(t^{\rho}-s^{\rho}\right)^{\alpha-1} \mathrm{~d} s \\
& \leq \frac{\|\psi\|_{0}}{\rho^{\alpha} \Gamma(\alpha+1)}\left(b^{\rho}-a^{\rho}\right)^{\alpha} .
\end{aligned}
$$

Remark 1 (see [7]). Let $\alpha, \beta, \rho>0$, then the following properties are satisfied:

(i) $I_{a^{+}}^{\alpha, \rho}(\psi+\xi)(t)=I_{a^{+}}^{\alpha, \rho} \psi(t)+I_{a^{+}}^{\alpha, \rho} \xi(t)$.

(ii) $I_{a^{+}}^{\alpha, \rho} I_{a^{+}}^{\beta, \rho} \psi(t)=I_{a^{+}}^{(\alpha+\beta), \rho} \psi(t)$.

(iii) For $0<\alpha<\beta$, we have

$$
\begin{aligned}
& \left({ }^{R L} D_{a^{+}}^{\alpha, \rho} I_{a^{+}}^{\alpha, \rho} \psi\right)(t)=\psi(t), \quad t \in(a, b], \\
& \left({ }^{R L} D_{a^{+}}^{\alpha, \rho} I_{a^{+}}^{\beta, \rho} \psi\right)(t)=\left(I_{a^{+}}^{(\beta-\alpha), \rho} \psi\right)(t), \quad t \in(a, b] .
\end{aligned}
$$

Remark 2. Let $n-1<\alpha \leq n \in \mathbb{N} \quad$ and $\quad \psi_{(1-\alpha), \rho} \in C^{n}$ $\left([a, b], \mathbb{R}^{d}\right)$, then we have, for $t \in(a, b]$,

$$
I_{a^{+}}^{\alpha, \rho R L} D_{a^{+}}^{\alpha, \rho} \psi(t)=\psi(t)-\sum_{k=1}^{n} \frac{\psi_{(n-\alpha), \rho}^{(n-k)}(a)}{\Gamma(\alpha-k+1)}\left(\frac{t^{\rho}-a^{\rho}}{\rho}\right)^{\alpha-k} .
$$

Remark 3. Let $n-1<\alpha \leq n \in \mathbb{N}$ and $\psi \in C^{n}\left([a, b], \mathbb{R}^{d}\right)$, then we have that, for $t \in(a, b]$,

$$
\begin{aligned}
& \left(I_{a^{+}}^{\alpha, \rho C} D_{a^{+}}^{\alpha, \rho} \psi\right)(t)=\psi(t)-\sum_{k=0}^{n-1} \frac{\psi^{(p, k)}(a)}{k !}\left(\frac{t^{\rho}-a^{\rho}}{\rho}\right)^{k}, \\
& \left({ }^{C} D_{a^{+}}^{\alpha, \rho} I_{a^{+}}^{\alpha, \rho} \psi\right)(t)=\psi(t),
\end{aligned}
$$

where $n=\alpha$.

Theorem 1 (see Theorem 8 in [10]). Let $p(t), q(t)$ be two integrable functions and $r(t)$ be a continuous function on $[a, b]$. Assume that $p$ and $q$ are nonnegative, and $r$ is nonnegative and nondecreasing. If

$$
p(t) \leq q(t)+r(t) \rho^{1-\alpha} \int_{a}^{t} s^{\rho-1}\left(t^{\rho}-s^{\rho}\right)^{\alpha-1} p(s) \mathrm{d} s, \quad \forall t \in[a, b],
$$

then

$$
\begin{aligned}
p(t) \leq & q(t)+\int_{a}^{t} \sum_{k=1}^{\infty} \frac{\rho^{1-k \alpha}(r(t) \Gamma(\alpha))^{k}}{\Gamma(k \alpha)} s^{\rho-1} \\
& \cdot\left(t^{\rho}-s^{\rho}\right)^{k \alpha-1} q(s) d s, \quad \forall t \in[a, b] .
\end{aligned}
$$

Furthermore, if the function $q$ is nondecreasing, then

$$
p(t) \leq q(t) E_{\alpha, 1}\left[r(t) \Gamma(\alpha)\left(\frac{t^{\rho}-a^{\rho}}{\rho}\right)^{\alpha}\right], \quad \forall t \in[a, b] .
$$

The existence and uniqueness results are proved according to the following Schauder's and Weissinger's fixed point theorem [31].

Theorem 2. Assume that $(X, d)$ is a complete metric space, and let $S$ be a closed convex subset of $X$. Furthermore, let $\mathbb{P}: S \longrightarrow S$ be the map such that the set $\{\mathbb{P} x: x \in S\}$ is relatively compact in $X$. Then, the operator $P$ has at least one fixed point $x^{*} \in S$ such that $\mathbb{P} x^{*}=x^{*}$.

Theorem 3. Assume that $(X, d)$ is a nonempty complete metric space and let $\lambda_{n} \geq 0$ for every $n \in \mathbb{N}_{0}$ such that the series $\sum_{n=0}^{\infty} \lambda_{n}$ converges. Furthermore, let the mapping $\mathbb{P}: X \longrightarrow X$ satisfy the inequality

$$
d\left(\mathbb{P}^{n} x, \mathbb{P}^{n} y\right) \leq \lambda_{n} d(x, y),
$$

for every $n \in \mathbb{N}$ and for any $x, y \in X$. Then, the operator $P$ has a unique fixed point $x_{0}$. Moreover, for any $x^{*} \in X$, the sequence $\left(\mathbb{P}^{n} x^{*}\right)_{n \geq 1}$ converges to the fixed point $x_{0}$.

\section{The Existence and Uniqueness of the Solution}

In this section, we reconsider the following fractional differential equations:

$$
\begin{aligned}
{ }^{C} D_{a^{+}}^{\alpha, \rho} \psi(t) & =f(t, \psi(t)), \\
\psi(a) & =\psi_{1}, \\
\psi^{\prime}(a) & =\psi_{2}, \\
t & \in[a, b] .
\end{aligned}
$$

A function $\psi:[a, b] \longrightarrow \mathbb{R}^{d}$ is said to be a solution of problem (16); if $\psi$ is continuous, $\psi(a)=\psi_{1}, \psi^{\prime}(a)=\psi_{2}$, and ${ }^{C} D_{a^{+}}^{\alpha, \rho} \psi(t)=f(t, \psi)$.

Theorem 4. Let the function $f(t, \psi)$ belong to $C_{\gamma}\left([a, b], \mathbb{R}^{d}\right)$, where $\gamma \in[0,1)$. Then, problem (16) is equivalent to the fractional integral equation: 


$$
\psi(t)=\psi_{0}+\frac{\left(t^{\rho}-a^{\rho}\right)}{\rho} \psi^{(\rho, 1)}(a)+\frac{1}{\Gamma(\alpha)} \int_{a}^{t} s^{\rho-1}\left(\frac{t^{\rho}-s^{\rho}}{\rho}\right)^{\alpha-1} f(s, \psi(s)) \mathrm{d} s, \quad t \in[a, b]
$$

where $\psi^{(\rho, 1)}(a):=\left[\left(t^{1-p} d / d t\right) \psi(t)\right]_{t=a}$.

Proof. Let $\psi \in C^{1}\left([a, b], \mathbb{R}^{d}\right)$ be a solution of $(16)$, then from (16) and Remark 3 we have that, for $\alpha \in(1,2)$,

$$
\left(I_{a^{+}}^{\alpha, \rho C} D_{a^{+}}^{\alpha, \rho} \psi\right)(t)=\psi(t)-\psi(a)-\frac{\left(t^{\rho}-a^{\rho}\right)}{\rho} \psi^{(\rho, 1)}(a),
$$

for $t \in[a, b]$. Because of the continuous hypotheses of the function $f$ and from (16), it yields that

$$
\begin{aligned}
\left(I_{a^{+}}^{\alpha, \rho C} D_{a^{+}}^{\alpha, \rho} \psi\right)(t)= & I_{a^{+}}^{\alpha, \rho} f(t, \psi(t))=\frac{1}{\Gamma(\alpha)} \int_{a}^{t} s^{\rho-1} \\
& \cdot\left(\frac{t^{\rho}-s^{\rho}}{\rho}\right)^{\alpha-1} f(s, \psi(s)) \mathrm{d} s, \quad t \in(a, b] .
\end{aligned}
$$

Consequently, by (18) and (3) we get the necessity condition. Conversely, let $\psi \in C^{1}\left([a, b], \mathbb{R}^{d}\right)$ satisfy the integral equation (17). Using the continuity of $f$ yields that $t \longmapsto f_{\alpha, \rho}(t, u):=I_{a^{+}}^{\alpha, \rho} f(t, u)$ is continuous on $(a, b]$ and $f_{\alpha, \rho}(a, u(a))=\lim _{t \longrightarrow a^{+}} f_{\alpha, \rho}(t, u)=0$. Indeed, it follows from the hypothesis $f \in C_{\gamma}\left([a, b], \mathbb{R}^{d}\right)$ that $\left(\left(t^{\rho}-a^{\rho}\right) / \rho\right)^{\gamma} f(t, u) \in C\left([a, b], \mathbb{R}^{d}\right)$ and, for all $t \in(a, b]$, there exists a positive constant $C$ such that

$$
\left\|\left(\frac{t^{\rho}-a^{\rho}}{\rho}\right)^{\gamma} f(t, u)\right\| \leq C, \quad \text { or } \quad f(t, u) \leq C\left(\frac{t^{\rho}-a^{\rho}}{\rho}\right)^{-\gamma} .
$$

This infers that

$$
\begin{gathered}
\left\|I_{a^{+}}^{\alpha, \rho} f(t, u)\right\| \leq I_{a^{+}}^{\alpha, \rho}\|f(t, u) \leq C I\|_{a^{+}}^{\alpha, \rho}\left(\frac{t^{\rho}-a^{\rho}}{\rho}\right)^{-\gamma} \\
=C \frac{\Gamma(1-\gamma)}{\Gamma(\alpha-\gamma+1)}\left(\frac{t^{\rho}-a^{\rho}}{\rho}\right)^{\alpha-\gamma} .
\end{gathered}
$$

Taking the limit when $t \longrightarrow a^{+}$, we observe that the right-hand side of (21) tends to 0 . Furthermore, $\psi(a)=\psi_{1}$ and $\psi^{\prime}(a)=\psi_{2}$. Next, by taking the Caputo-Katugampola fractional derivative, ${ }^{R L} D_{a^{+}}^{\alpha, \rho}$, on the two sides of (17) and by Remark 1 one has that

$$
{ }^{C} D_{a^{+}}^{\alpha, \rho} \psi(t)=f(t, \psi(t)), \quad t \in(a, b] .
$$

In the below theorems, we will present the existence and uniqueness of the local solution to problem (16) by using the Schauder fixed point theorem. We set $I(t):=\psi_{0}+\psi^{(\rho, 1)}(a)\left(\left(t^{\rho}-a^{\rho}\right) / \rho\right)$. Let $r>0$ be a given constant, and define. $\Omega:=\{(t, \psi) \in \quad[a, b] \times C$ $\left.\left([a, b], \mathbb{R}^{d}\right): \sup _{t \in[a, b]}\|\psi(t)-I(t)\| \leq r\right\}$.

Theorem 5. Let $f:[a, b] \times \mathbb{R}^{d} \longrightarrow \mathbb{R}^{d}$ be a continuous function and $\sup _{(t, \psi) \in \Omega}\|f(t, \psi)\| \leq M$. Then, problem (16) has at least one solution on $[a, b]$. Furthermore, if the following Lipschitz condition is held,

$$
\|f(t, \psi)-f(t, \xi)\| \leq L\|\psi-\xi\|,
$$

where $L$ is a positive constant, then problem (16) has a unique solution on $[a, b]$.

Proof. Let

$$
\Omega_{T}=\left\{\psi: \psi \in C\left([a, T], \mathbb{R}^{d}\right), \sup _{t \in[a, T]}\|\psi(t)-I(t)\| \leq r\right\},
$$

where

$$
T=\min \left\{b,\left(\left(r \frac{\rho^{\alpha} \Gamma(1+\alpha)}{M}\right)^{1 / \alpha}+a^{\rho}\right)^{1 / \rho}\right\}
$$

We observe that $\Omega_{T} \subseteq C\left([a, T], \mathbb{R}^{d}\right)$ is nonempty, bounded, closed, and convex subset. Define the operator $\mathbb{T}: \Omega_{T} \longrightarrow\left[\psi \mid \psi \in C\left([a, T], \mathbb{R}^{d}\right)\right]$ by

$$
\left(\mathbb{\psi} \psi(t)=I(t)+\frac{1}{\Gamma(\alpha)} \int_{a}^{t} s^{\rho-1}\left(\frac{t^{\rho}-s^{\rho}}{\rho}\right)^{\alpha-1} f(s, \psi(s)) \mathrm{d} s .\right.
$$

The proof of this theorem is divided into two steps. In the first step, we shall prove that the operator $\mathrm{T}$ has at least one fixed point by using Schauder's fixed point principle and in the second one we also verify that the operator $\mathbb{T}$ has a unique solution by using Weissinger's fixed point theorem.

Step 1. We shall show that the conditions of Theorem 2 are satisfied.

We show that $\mathbb{Z} \Omega_{T} \subset \Omega_{T}$. For any $\psi \in \Omega_{T}$, we obtain

$$
\begin{aligned}
\|(\mathbb{W} \psi)(t)-I(t)\| & \leq \frac{1}{\Gamma(\alpha)} \int_{a}^{t} s^{\rho-1}\left(\frac{t^{\rho}-s^{\rho}}{\rho}\right)^{\alpha-1}\|f(s, \psi(s))\| \mathrm{d} s \\
& \leq \frac{M}{\Gamma(\alpha+1)}\left(\frac{t^{\rho}-a^{\rho}}{\rho}\right)^{\alpha} \leq r .
\end{aligned}
$$

On the other hand, let $\psi, \psi_{n} \in \Omega_{T}$ such that $\psi_{n} \longrightarrow \psi$ in $C\left([a, T], \mathbb{R}^{d}\right)$ as $n \longrightarrow \infty$. By the continuity of the function $f$ one has $f\left(t, \psi_{n}\right) \longrightarrow f(t, \psi)$ in $C\left([a, T], \mathbb{R}^{d}\right)$ as $n \longrightarrow \infty$. Since

$$
\begin{aligned}
\left\|\left(\mathbb{T} \psi_{n}\right)(t)-(\mathbb{T} \psi)(t)\right\| \leq & \frac{1}{\Gamma(\alpha)} \int_{a}^{t} s^{\rho-1}\left(\frac{t^{\rho}-s^{\rho}}{\rho}\right)^{\alpha-1} \\
& \cdot \sup _{s \in[a, t]}\left\|f\left(s, \psi_{n}(s)\right)-f(s, \psi(s))\right\| \mathrm{d} s,
\end{aligned}
$$

we have $\quad \sup _{t \in[a, T]}\left\|\left(\mathbb{\nabla} \psi_{n}\right)(t)-(\mathbb{\nabla} \psi)(t)\right\| \leq$ $\left(\rho^{-\alpha} / \Gamma(\alpha+1)\right)\left(t^{\rho}-a^{\rho}\right)^{\alpha} \sup _{s \in[a, t]}\left\|f\left(s, \psi_{n}(s)\right)-f(s, \psi(s))\right\|$. Then, $\left(\mathbb{T} \psi_{n}\right)(t)$ tends to $(\mathbb{T} \psi)(t)$ in $\Omega_{T}$ as $n \longrightarrow \infty$. This 
yields $\mathbb{T}$ is continuous. Thus, we infer that $\mathbb{\psi} \psi \in \Omega_{T}$, for $\psi \in \Omega_{T}$, i.e., $\mathbb{T} \Omega_{T} \subseteq \Omega_{T}$.

Next, we show that $\mathbb{T} \Omega_{T}=\left\{(\mathbb{\psi} \psi)(t): \psi \in \Omega_{T}\right\}$ is a relatively compact set. For $\mathbb{\psi} \psi \in \mathbb{T} \Omega_{T}$ and $t \in[a, T]$, since

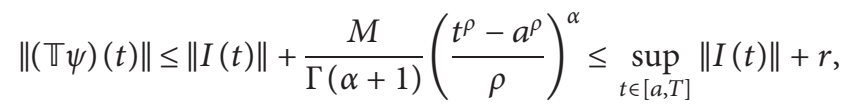

we conclude that the set $T \Omega_{T}$ is uniformly boundedness. Furthermore, for $a \leq t_{1} \leq t_{2} \leq T$

$$
\|\left(\mathbb{W} \psi\left(t_{1}\right)-(\mathbb{T} \psi)\left(t_{2}\right)+I\left(t_{2}\right)-I\left(t_{1}\right) \| \leq \frac{M \rho^{-\alpha}}{\Gamma(\alpha+1)}\left|t_{2}^{\rho}-t_{1}^{\rho}\right|^{\alpha}+\frac{M \rho^{-\alpha}}{\Gamma(\alpha+1)}\left(\left|t_{2}^{p \alpha}-t_{1}^{p \alpha}\right|-\left|t_{2}^{\rho}-t_{1}^{\rho}\right|^{\alpha}\right) \leq \frac{M \rho^{-\alpha}}{\Gamma(\alpha+1)}\left(\left(t_{2}^{\rho}-t_{1}^{\rho}\right)^{\alpha}+\left(t_{2}^{p \alpha}-t_{1}^{p \alpha}\right)\right) .\right.
$$

Applying the mean value theorem, one obtains

$$
\|\left(\mathbb{\psi} \psi\left(t_{1}\right)-(\mathbb{\psi} \psi)\left(t_{2}\right) \| \leq\left(t_{2}-t_{1}\right) \tau^{\rho-1}+\frac{M \rho^{-\alpha}}{\Gamma(\alpha+1)}\left(\tau^{\alpha(\rho-1)}\left(\frac{t_{2}-t_{1}}{\rho}\right)^{\alpha}+\alpha \tau^{\rho-1}\left(\frac{t_{2}-t_{1}}{\rho}\right)\right),\right.
$$

for some $\tau \in\left[t_{1}, t_{2}\right] \subseteq[a, T]$. Therefore, if $\left|t_{2}-t_{1}\right|<\delta$, we have

$$
\begin{aligned}
\|(\mathbb{\psi} \psi)\left(t_{1}\right)-\left(\mathbb{\psi} \psi\left(t_{2}\right) \| \leq\right. & M \frac{\delta \tau^{\rho-1}}{\Gamma(\alpha+1)}\left(\tau^{\alpha(\rho-1)} \delta^{\alpha}+\rho^{1-\alpha} \alpha \tau^{\rho-1} \delta\right) \leq \max \left\{a^{\rho-1}, T^{\rho-1}\right\} \delta+\frac{M}{\Gamma(\alpha+1)}\left(\max \left\{a^{\alpha(\rho-1)}, T^{\alpha(\rho-1)}\right\} \delta^{\alpha}\right. \\
& \left.+\rho^{1-\alpha} \alpha \max \left\{a^{\rho \alpha-1}, T^{\rho \alpha-1}\right\} \delta\right),
\end{aligned}
$$

where $a>0, \rho>0$ is fixed, and $\alpha \in(1,2)$. This shows that $\mathbb{} \mathbb{} \Omega_{T}$ is equicontinuous. Hence, by Arzela-Ascoli theorem (see Theorem 1.8 in [5]), this yields that $\mathrm{T} \Omega_{T}$ is relatively compact. So, according to the conditions of Theorem 2, we can conclude that the operator $\mathrm{T}$ has a fixed point.

Step 2. For the uniqueness of solution, we suppose that $\xi(\cdot):[a, T] \longrightarrow C\left([a, T], \mathbb{R}^{d}\right)$ is another solution for problem (16) on $[a, T]$ and $\xi(a)=\psi(a), \xi^{\prime}(a)=\psi^{\prime}(a)$. Then, we obtain

$\|(\mathbb{T} \psi)(t)-(\mathbb{\nabla} \xi)(t)\| \leq \frac{L}{\Gamma(\alpha)} \int_{a}^{t} s^{\rho-1}\left(\frac{t^{\rho}-s^{\rho}}{\rho}\right)^{\alpha-1}\|\psi(s)-\xi(s)\| \mathrm{d} s$.

Thus, it follows that

$$
\begin{aligned}
& \sup _{t \in[a, T]}\|(\mathbb{\psi} \psi)(t)-(\mathbb{T} \xi)(t)\| \leq \frac{L}{\Gamma(\alpha+1)}\left(\frac{t^{\rho}-a^{\rho}}{\rho}\right)^{\alpha} \\
& \text {. } \sup _{t \in[a, T]}\|\psi(s)-\xi(s)\| .
\end{aligned}
$$

By the induction method, we will verify that

$$
\begin{aligned}
\sup _{t \in[a, T]}\left\|\left(\mathbb{T}^{n} \psi\right)(t)-\left(\mathbb{T}^{n} \xi\right)(t)\right\| \leq & \frac{L^{n}}{\Gamma(n \alpha+1)}\left(\frac{t^{\rho}-a^{\rho}}{\rho}\right)^{n \alpha} \\
& \cdot \sup _{t \in[a, T]}\|\psi(t)-\xi(t)\|,
\end{aligned}
$$

where

$$
\left(\mathbb{T}^{n} \psi\right)(t)=I(t)+\frac{1}{\Gamma(\alpha)} \int_{a}^{t} s^{\rho-1}\left(\frac{t^{\rho}-s^{\rho}}{\rho}\right)^{\alpha-1}
$$

$$
\cdot f\left(s,\left(\mathbb{T}^{n-1} \psi\right)(s)\right) \mathrm{d} s
$$

for $n \in \mathbb{N}$. Indeed, we assume that (35) is satisfied for the case of $(n-1) \in \mathbb{N}$, and for $n \in \mathbb{N}$, one has that 


$$
\begin{aligned}
\sup _{t \in[a, T]}\left\|\left(\mathbb{T}^{n} \psi\right)(t)-\left(\mathbb{T}^{n} \xi\right)(t)\right\| & =\sup _{t \in[a, T]} \|\left(\mathbb{T}\left(\mathbb{T}^{n-1} \psi\right)\right)(t)-\left(\mathbb{T}\left(\left(\mathbb{T}^{n-1} \xi\right)\right)(t) \|\right. \\
& \leq \frac{L}{\Gamma(\alpha+1)} \int_{a}^{t} s^{\rho-1}\left(\frac{t^{\rho}-s^{\rho}}{\rho}\right)^{\alpha-1} \sup _{s \in[a, T]}\left\|\left(\mathbb{T}^{n-1} \psi\right)(s)-\left(\mathbb{T}^{n-1} \xi\right)(s)\right\| \mathrm{d} s \\
& \leq \frac{\rho^{1-n \alpha} L^{n}}{\Gamma((n-1) \alpha+1)} \sup _{t \in[a, T]}\|\psi(t)-\xi(t)\| \frac{1}{\Gamma(\alpha+1)} \int_{a}^{t} s^{\rho+\rho \alpha(n-1)-1}\left(t^{\rho}-s^{\rho}\right)^{\alpha-1} \mathrm{~d} s \\
& =\frac{L^{n}}{\Gamma(n \alpha+1)}\left(\frac{t^{\rho}-a^{\rho}}{\rho}\right)^{n \alpha} \sup _{t \in[a, T]}\|\psi(t)-\xi(t)\| .
\end{aligned}
$$

Setting $K_{n}:=\left(L^{n} / \Gamma(n \alpha+1)\right)\left(\left(t^{\rho}-a^{\rho}\right) / \rho\right)^{n \alpha}$, we observe that the series $\sum_{n=0}^{\infty} K_{n}$ converges to the Mittag-Leffler function $E_{\alpha, 1}\left(L\left(\left(t^{\rho}-a^{\rho}\right) / \rho\right)^{\alpha}\right)$. So, we can conclude that the series $K_{n}$ is convergent. Based on Theorem 3 , the operator T has a unique fixed point according to the conditions of Weissinger's fixed point theorem.

Theorem 6. Assume that the function $f:[a, b] \times \mathbb{R}^{d} \longrightarrow \mathbb{R}^{d}$ is a continuous function and $\sup _{(t, \psi) \in \Omega}\|f(t, \psi)\| \leq M$. Let $\psi(t)$ be a solution of problem (16) on $[a, \beta)$. If $\psi(t)$ is bounded on $[\eta, \beta)$ for some $\eta>a$, then $\beta=+\infty$.

Proof. This assertion will be divided into two steps. First of all, we verify $\lim _{t \longrightarrow \beta^{-}} \psi(t)$ exists. It means there exists $\eta \in(a, \beta)$ such that, for $t \in(\eta, \beta),\left\|\psi(t)-\psi^{*}\right\|<\varepsilon$, for any given $\varepsilon>0$, i.e.,

$$
\lim _{t \longrightarrow \beta^{-}} \psi(t)=\psi^{*}
$$

Since $\psi(t)$ is bounded on $[\eta, \beta)$, there exists a sequence $\left\{t_{k}\right\}$ and a positive constant $K>0$ such that $t_{k}<t_{k+1}, k \in \mathbb{N}$, $\lim _{k \rightarrow \infty} t_{k}=\beta$, and $\left\|\psi\left(t_{k}\right)\right\| \leq K$. In addition, as $\left\{\psi\left(t_{k}\right)\right\}$ is a bounded convergent subsequence, it follows that $\lim _{k \longrightarrow \infty} \psi\left(t_{k}\right)=\psi^{*}$. This implies that there exists $k_{0}$ such that $t_{k_{0}}>\eta$, and for $k \geq k_{0}$, we have

$$
\left\|\psi\left(t_{k}\right)-\psi^{*}\right\| \leq \frac{\varepsilon}{2}
$$

If (38) is not true, then for $k \geq k_{0}$, there exists $\tau_{k} \in\left(t_{k}, \beta\right)$ such that $\left\|\psi\left(\tau_{k}\right)-\psi^{*}\right\| \geq \varepsilon$ and $\left\|\psi(t)-\psi^{*}\right\|<\varepsilon, t \in\left(t_{k}, \tau_{k}\right)$. Then, from (39) and triangle inequality one has

$$
\begin{aligned}
\varepsilon \leq & \left\|\psi\left(t_{k}\right)-\psi^{*}\right\|+\left\|\psi\left(\tau_{k}\right)-\psi\left(t_{k}\right)\right\| \\
\leq & \frac{\varepsilon}{2}+\left\|\frac{\psi^{(\rho, 1)}(a)}{\rho}\left(\tau_{k}^{\rho}-t_{k}^{\rho}\right)\right\|+\frac{1}{\Gamma(\alpha)} \int_{a}^{t_{k}} s^{\rho-1}\left[\left(\frac{\tau_{k}^{\rho}-s^{\rho}}{\rho}\right)^{\alpha-1}-\left(\frac{t_{k}^{\rho}-s^{\rho}}{\rho}\right)^{\alpha-1}\right]\|f(s, \psi(s))\| \mathrm{d} s \\
& +\frac{1}{\Gamma(\alpha)} \int_{t_{k}}^{\tau_{k}} s^{\rho-1}\left(\frac{t_{k}^{\rho}-s^{\rho}}{\rho}\right)^{\alpha-1}\|f(s, \psi(s))\| \mathrm{d} s \\
\leq & \frac{\varepsilon}{2}+\left\|\frac{\psi^{(\rho, 1)}(a)}{\rho}\left(\tau_{k}^{\rho}-t_{k}^{\rho}\right)\right\|+\frac{M}{\rho^{\alpha} \Gamma(\alpha+1)}\left(\left(\tau_{k}^{\rho}-t_{k}^{\rho}\right)^{\alpha}+\left(\tau_{k}^{p \alpha}-t_{k}^{p \alpha}\right)\right) .
\end{aligned}
$$

Then, for sufficiently large $k \geq k_{0}$, we have

$$
\varepsilon \leq\left\|\psi\left(\tau_{k}\right)-\psi^{*}\right\|<(\varepsilon / 2)+(\varepsilon / 2)=\varepsilon .
$$

This implies the contradiction that $\lim _{t \longrightarrow \beta^{-}} \psi(t)$ exists. For the next step, we show that $\psi(t)$ is continuable. Define $\psi(\beta)=\lim _{t \longrightarrow \beta^{-}} \psi(t)$. Then, $\psi(t)$ is continuous on $[a, \beta]$. Consider the operator $\mathbb{T}$ as follows:

$$
\begin{aligned}
(\mathbb{\psi} \psi(t)= & I(t)+\frac{1}{\Gamma(\alpha)} \int_{a}^{\beta} s^{\rho-1}\left(\frac{t^{\rho}-s^{\rho}}{\rho}\right)^{\alpha-1} f(s, \psi(s)) \mathrm{d} s \\
& +\frac{1}{\Gamma(\alpha)} \int_{\beta}^{t} s^{\rho-1}\left(\frac{t^{\rho}-s^{\rho}}{\rho}\right)^{\alpha-1} f(s, \psi(s)) \mathrm{d} s
\end{aligned}
$$

where $\psi \in C\left([\beta, \beta+1], \mathbb{R}^{d}\right), t \in[\beta, \beta+1]$. As a result of Theorem 5 , since on the interval $[a, \beta]$, equation (42) 
possesses a solution, and we can rewrite equation (42) as follows:

$$
(\mathbb{\psi} \psi)(t)=\psi_{1}(t)+\frac{1}{\Gamma(\alpha)} \int_{\beta}^{t} s^{\rho-1}\left(\frac{t^{\rho}-s^{\rho}}{\rho}\right)^{\alpha-1} f(s, \psi(s)) \mathrm{d} s,
$$

where

$$
\psi_{1}(t)=I(t)+\frac{1}{\Gamma(\alpha)} \int_{a}^{\beta} s^{\rho-1}\left(\frac{t^{\rho}-s^{\rho}}{\rho}\right)^{\alpha-1} f(s, \psi(s)) \mathrm{d} s,
$$

is the known function. Let $\Omega^{*}=\{(t, \psi): \beta \leq$ $\left.t \leq \beta+1,\|\psi\| \leq \sup _{t \in[\beta, \beta+1]}\left\|\psi_{1}(t)\right\|+\rho_{1}\right\}$. Because of the continuation of $f$ on $\Omega^{*}$, denote $M^{*}=\max _{(t, \psi) \in \Omega^{*}}\|f(t, \psi)\|$. Again let

$$
\begin{aligned}
\Omega_{h}= & \left\{\psi: \psi \in C\left([\beta, \beta+1], \mathbb{R}^{d}\right), \sup _{t \in[\beta, \beta+h]}\left\|\psi(t)-\psi_{1}(t)\right\|\right. \\
& \left.\leq \rho_{1}, \psi(\beta)=\psi_{1}(\beta)\right\},
\end{aligned}
$$

$$
h=\min \left\{1,\left(\left(\rho_{1} \frac{\rho^{\alpha} \Gamma(1+\alpha)}{M^{*}}\right)^{1 / \alpha}+\beta^{\rho}\right)^{1 / p}\right\} .
$$

By the same argument as in Theorem 5, we show that $\mathbb{T}$ has a fixed point. Let $\left\{\psi_{n}\right\} \subseteq C\left([\beta, \beta+h], \mathbb{R}^{d}\right)$, such that $\psi_{n} \longrightarrow \psi$ in $C\left([\beta, \beta+h], \mathbb{R}^{d}\right)$ as $n \longrightarrow \infty$. By the continuity hypothesis of the function $f$ we have $f\left(t, \psi_{n}\right) \longrightarrow f(t, \psi)$ in $C\left([\beta, \beta+h], \mathbb{R}^{d}\right)$ as $n \longrightarrow \infty$. Since

$$
\begin{aligned}
\left\|\left(\mathbb{\nabla} \psi_{n}\right)(t)-(\mathbb{T} \psi)(t)\right\| \leq & \frac{h^{p \alpha}}{\rho^{\alpha} \Gamma(\alpha+1)} \sup _{s \in[\beta, \beta+h]} \| f\left(s, \psi_{n}(s)\right) \\
& -f(s, \psi(s)) \|,
\end{aligned}
$$

we have $\sup _{t \in[\beta, \beta+h]}\left\|\left(\mathbb{T} \psi_{n}\right)(t)-(\mathbb{\nabla} \psi)(t)\right\| \longrightarrow 0$ as $n \longrightarrow \infty$, which yields that operator $\mathbb{\mathbb { T }}$ is continuous. Next, it follows that $\mathbb{T} \Omega_{h}=\left\{\left(\mathbb{\psi} \psi(t): \psi \in \Omega_{h}\right\}\right.$ is relatively compact. For $\mathbb{\psi} \in \mathbb{\square} \Omega_{h}$ and $t \in[\beta, \beta+h]$, we have

$$
\left\|(\mathbb{\psi} \psi)(t)-\psi_{1}\right\| \leq \frac{M_{1}\left(t^{\rho}-\beta^{\rho}\right)^{\alpha}}{\rho^{\alpha} \Gamma(\alpha+1)} \leq \rho_{1} .
$$

Thus, $\quad \mathbb{} \quad \Omega_{h} \subset \Omega_{h} . \quad$ Furthermore, for $\psi \in \Omega_{h}, \beta \leq t_{1} \leq t_{2} \leq \beta+h$, one has

where

$$
\begin{aligned}
\left\|(\mathbb{\psi} \psi)\left(t_{1}\right)-(\mathbb{T} \psi)\left(t_{2}\right)+\psi_{1}\left(t_{2}\right)-\psi_{1}\left(t_{1}\right)\right\| \leq & \frac{\rho^{1-\alpha}}{\Gamma(\alpha)} \int_{\beta}^{t_{1}} s^{\rho-1}\left(\left(t_{2}^{\rho}-s^{\rho}\right)^{\alpha-1}-\left(t_{1}^{\rho}-s^{\rho}\right)^{\alpha-1}\right)\|f(s, \psi(s))\| \mathrm{d} s \\
& +\frac{\rho^{1-\alpha}}{\Gamma(\alpha)} \int_{t_{1}}^{t_{2}} s^{\rho-1}\left(t_{2}^{\rho}-s^{\rho}\right)^{\alpha-1}\|f(s, \psi(s))\| \mathrm{d} s \\
\leq & \frac{M^{*} \rho^{-\alpha}}{\Gamma(\alpha+1)}\left(\left(t_{2}^{\rho}-t_{1}^{\rho}\right)^{\alpha}+\left(t_{2}^{p \alpha}-t_{1}^{p \alpha}\right)\right) .
\end{aligned}
$$

With the same argument as in the proof of Theorem 5 , since $\psi_{1}(t)$ is continuous on $[\beta, \beta+1]$, it follows that $\mathbb{T} \Omega_{h}$ is equicontinuous. Hence, by Arzela-Ascoli theorem, we deduce that $\mathbb{U} \Omega_{h}$ is relatively compact. Therefore, by the Schauder's fixed point theorem, operator $\mathbb{T}$ has a fixed point $\widehat{\psi}(t) \in \Omega_{h}$, i.e.,

$$
\begin{aligned}
\widehat{\psi}(t) & =\psi_{1}(t)+\frac{1}{\Gamma(\alpha)} \int_{\beta}^{t} s^{\rho-1}\left(\frac{t^{\rho}-s^{\rho}}{\rho}\right)^{\alpha-1} f(s, \widehat{\psi}(s)) \mathrm{d} s \\
& =I(t)+\frac{1}{\Gamma(\alpha)} \int_{a}^{t} s^{\rho-1}\left(\frac{t^{\rho}-s^{\rho}}{\rho}\right)^{\alpha-1} f(s, \widehat{\psi}(s)) \mathrm{d} s,
\end{aligned}
$$

$t \in[\beta, \beta+h]$, where

$$
\widehat{\psi}(t)= \begin{cases}\psi(t), & t \in(a, \beta], \\ \widehat{\psi}(t), & t \in[\beta, \beta+h] .\end{cases}
$$

According to Theorem 4 , we conclude that $\widehat{\psi}(t)$ is a solution of problem (16) on $(a, \beta+h]$. By the assumption of this theorem again, it follows that $\psi(t)$ can be extended beyond $\beta$. So, the solution of problem (16) exists on $[a, \infty)$ and so $\beta=+\infty$.
Theorem 7. Let $f:[a,+\infty) \mathbb{R}^{d} \longrightarrow \mathbb{R}^{d}$ be a continuous function and there exists a continuous function $q(t):[a,+\infty) \longrightarrow[0,+\infty)$ such that $\|f(t, \psi)\| \leq$ $M\|\psi\|+q(t)$, where $M>0$ is a constant. Then, problem (16) has at least one solution on $[a,+\infty)$. Furthermore, if the following Lipschitz condition is satisfied,

$$
\|f(t, \psi)-f(t, \xi)\| \leq L\|\psi-\xi\|, \quad \forall t \in[a,+\infty),
$$

then problem (16) has a unique solution on $[a,+\infty)$.

Proof. It follows from the assertion of Theorem 5 that there exists a solution $\psi(t)$ of problem (16). By Theorem 4, $\psi(t)$ satisfies the following integral equation

$$
\begin{aligned}
\psi(t)= & \psi_{0}+\frac{\left(t^{\rho}-a^{\rho}\right)}{\rho} \psi^{(\rho, 1)}(a)+\frac{1}{\Gamma(\alpha)} \int_{a}^{t} s^{\rho-1}\left(\frac{t^{\rho}-s^{\rho}}{\rho}\right)^{\alpha-1} \\
& \cdot f(s, \psi(s)) \mathrm{d} s .
\end{aligned}
$$

Step 3 (the existence of solution). Suppose that the solution $\psi$ admits a maximal existence interval, denoted by $[a, \beta)$ 
$(\beta<+\infty)$. By the condition of the function $f$, one has the estimate

$$
\begin{gathered}
\|\psi(t)\| \leq\left\|\psi_{0}+\frac{\psi^{(\rho, 1)}(a)}{\rho}\left(t^{\rho}-a^{\rho}\right)\right\|+\frac{1}{\Gamma(\alpha)} \int_{a}^{t} s^{\rho-1} \\
\cdot\left(\frac{t^{\rho}-s^{\rho}}{\rho}\right)^{\alpha-1}(M\|\psi(s)\|+q(s)) \mathrm{d} s .
\end{gathered}
$$

By putting $\quad v(t)=\| \psi_{0}+\left(\psi^{(\rho, 1)}(a) / \rho\right)$ $\left(t^{\rho}-a^{\rho}\right) \|+\left(\rho^{1-\alpha} / \Gamma(\alpha)\right) \int_{a}^{t} s^{\rho-1}\left(t^{\rho}-s^{\rho}\right)^{\alpha-1} q(s) \mathrm{d} s$., then (54) leads to

$$
\|\psi(t)\| \leq v(t)+\frac{M \rho^{1-\alpha}}{\Gamma(\alpha)} \int_{a}^{t} s^{\rho-1}\left(t^{\rho}-s^{\rho}\right)^{\alpha-1}\|\psi(s)\| \mathrm{d} s .
$$

We now prove that $w(t):=\|\psi(t)\|$ is bounded on $[a, \beta)$. Define the functional

$$
\mathscr{F} w(t)=\frac{M \rho^{1-\alpha}}{\Gamma(\alpha)} \int_{a}^{t} s^{\rho-1}\left(t^{\rho}-s^{\rho}\right)^{\alpha-1} w(s) \mathrm{d} s .
$$

Then, one has that $w(t) \leq v(t)+\mathscr{F} w(t)$, and by iterating consecutively, we also obtain for $n \in \mathbb{N}$,

$$
w(t) \leq \sum_{i=0}^{n-1} \mathscr{F}^{i} v(t)+\mathscr{F}^{n} w(t)
$$

In addition, by mathematical induction and $v(t)>0$ for $t \in[a, \beta)$, then (56) leads to the following estimation:

$$
\mathscr{F}^{i} v(t) \leq \frac{M^{i}}{\Gamma(i \alpha)} \int_{a}^{t} s^{\rho-1}\left(\frac{t^{\rho}-s^{\rho}}{\rho}\right)^{i \alpha-1} v(s) \mathrm{d} s, \quad i \in \mathbb{N} .
$$

Indeed, for $i=1,(58)$ is obvious. If (58) is valid for $i \in \mathbb{N}$, then by using Dirichlet's formula, we obtain

$$
\begin{aligned}
\mathscr{F}^{i+1} v(t)=\mathscr{F} \mathscr{F}^{i} v(t) & \leq \frac{M \rho^{1-\alpha}}{\Gamma(\alpha)} \int_{a}^{t} s^{\rho-1}\left(t^{\rho}-s^{\rho}\right)^{\alpha-1}\left(\frac{M^{i}}{\Gamma(i \alpha)} \int_{a}^{s} \tau^{p-1}\left(\frac{s^{\rho}-\tau^{\rho}}{\rho}\right)^{i \alpha-1} v(\tau) \mathrm{d} \tau\right) \mathrm{d} s \\
& =\frac{M^{i+1} \rho^{2-(i+1) \alpha}}{\Gamma(\alpha) \Gamma(i \alpha)} \int_{a}^{t} \int_{a}^{s} s^{\rho-1}\left(t^{\rho}-s^{\rho}\right)^{\alpha-1} \tau^{p-1}\left(s^{\rho}-\tau^{\rho}\right)^{i \alpha-1} v(\tau) \mathrm{d} \tau \mathrm{d} s \\
& =\frac{M^{i+1} \rho^{2-(i+1) \alpha}}{\Gamma(\alpha) \Gamma(i \alpha)} \int_{a}^{t} s^{\rho-1} v(s) \int_{s}^{t} \tau^{p-1}\left(t^{\rho}-\tau^{\rho}\right)^{\alpha-1}\left(\tau^{\rho}-s^{\rho}\right)^{i \alpha-1} \mathrm{~d} \tau \mathrm{d} s .
\end{aligned}
$$

With the change of variables $z=\left(s^{\rho}-a^{\rho}\right) /\left(t^{\rho}-a^{\rho}\right)$, we obtain

$$
\mathscr{F}^{i+1} v(t) \leq \frac{M^{i+1}}{\Gamma(i \alpha+\alpha)} \int_{a}^{t} s^{\rho-1}\left(\frac{t^{\rho}-s^{\rho}}{\rho}\right)^{(i+1) \alpha-1} v(s) \mathrm{d} s .
$$

Thus, (58) is valid for $i+1$. Besides, we observe that $\mathscr{F}^{n} w(t) \longrightarrow 0$ as $n \longrightarrow \infty$. Hence, from (57), we have

$$
w(t) \leq \sum_{i=0}^{\infty} \mathscr{F}^{i} v(t) \leq v(t)+\int_{a}^{t} \sum_{i=1}^{\infty} \frac{M^{i}}{\Gamma(i \alpha)} s^{\rho-1}\left(\frac{t^{\rho}-s^{\rho}}{\rho}\right)^{i \alpha-1} v(s) \mathrm{d} s .
$$

Furthermore, if the function $v$ is nondecreasing, then for all $s \in[a, t]$, we have $v(s) \leq v(t)$ and so

$$
\begin{aligned}
w(t) & \leq v(t)\left[1+\sum_{i=1}^{\infty} \frac{M^{i}}{\Gamma(i \alpha)} \int_{a}^{t} s^{\rho-1}\left(\frac{t^{\rho}-s^{\rho}}{\rho}\right)^{i \alpha-1} \mathrm{~d} s\right] \\
& =v(t) E_{\alpha, 1}\left(M\left(\frac{t^{\rho}-a^{\rho}}{\rho}\right)^{\alpha}\right) .
\end{aligned}
$$

Therefore, we deduce that $\psi(t)$ is bounded on $[a, \beta)$. It follows from Theorem 6 that the solution $\psi(t)$ can be extended to the right side of $\beta$. This obviously contradicts the assumption that $[a, \beta)$ is the maximal existence interval. This consequently implies that $\beta=+\infty$.
Step 4 (the uniqueness of solution). By the Lipschitz condition and inequality (62) the uniqueness of solution can be proved. Indeed, let us assume that $\xi(t)$ is another solution for problem (16) and $\xi(a)=\psi(a), \xi^{\prime}(a)=\psi \prime(a)$. Then, one has that

$$
\begin{aligned}
\|\psi(t)-\xi(t)\| \leq & \left\|\psi_{0}(t)-\xi_{0}(t)\right\|+\frac{L}{\Gamma(\alpha)} \int_{a}^{t} s^{\rho-1}\left(\frac{t^{\rho}-s^{\rho}}{\rho}\right)^{\alpha-1} \\
\cdot & \|\psi(s)-\xi(s)\| \mathrm{d} s,
\end{aligned}
$$

where $\psi_{0}(t):=\psi(a)+\psi^{(\rho, 1)}(a)\left(\left(t^{\rho}-a^{\rho}\right) / \rho\right)$. Define $k(t)=$ $\|\psi(t)-\xi(t)\|$, and note that since $\xi(a)=$ $\psi(a), \xi^{\prime}(a)=\psi^{\prime}(a)$, we have $\left\|\psi_{0}(t)-\xi_{0}(t)\right\|=0$. Applying Theorem 1 , we get $k(t) \leq 0$. This implies that $\psi(t)=\xi(t)$, which completes the proof.

\section{The Stability of Problem (16)}

In the sequel, some kinds of the Ulam-Hyers-Mittag-Leffler stability for problem (16) will be investigated. Denote $J:=[a, b]$. Let $\alpha \in(1,2), \rho>0, \varepsilon>0$, and $\varphi \in C\left([a, b], \mathbb{R}^{+}\right)$. We consider the inequalities as follows:

$$
\begin{aligned}
& \left\|{ }^{C} D_{a^{+}}^{\alpha, \rho} \xi(t)-f(t, \xi(t))\right\| \leq \varepsilon, \\
& \left\|{ }^{C} D_{a^{+}}^{\alpha, \rho} \xi(t)-f(t, \xi(t))\right\| \leq \varepsilon \varphi(t) .
\end{aligned}
$$


Remark 4. From inequalities (64) and (65), we observe that

(i) If a function $\xi$ is a solution of (64), then there exists a function $\delta_{1} \in C\left(J, \mathbb{R}^{d}\right)$ such that $\left\|\delta_{1}\right\| \leq \varepsilon$, for all $t \in J$, and ${ }^{C} D_{a^{+}}^{\alpha, \rho} \xi(t)=f(t, \xi(t))+\delta_{1}(t)$

(ii) If a function $\xi$ is a solution of (65), then there exists a function $\delta_{2} \in C\left(J, \mathbb{R}^{d}\right)$ such that $\left\|\delta_{2}\right\| \leq \varepsilon \varphi(t)$, for all $t \in J$, and ${ }^{C} D_{a^{+}}^{\alpha, \rho} \xi(t)=f(t, \xi(t))+\delta_{2}(t)$

Definition 1. Let $\beta_{f} \geq 0$. roblem (16) is said to be

(i) Ulam-Hyers-Mittag-Leffler stable if there is a constant $C_{f}>0$ such that, for each $\varepsilon>0$ and for each solution $\xi$ of (64), there exists a solution $\psi$ of problem (16) satisfying the estimate

$$
\|\psi(t)-\xi(t)\| \leq \varepsilon C_{f} E_{\alpha, 1}\left(\beta_{f}\left(\frac{t^{\rho}-a^{\rho}}{\rho}\right)^{\alpha}\right), \quad t \in[a, b] .
$$

(ii) Ulam-Hyers-Rassias-Mittag-Leffler stable with respect to $(\varphi, \eta)$ if there is a constant $C_{\varphi}>0$ such that, for each $\varepsilon>0$ and for each solution $\xi$ of (65), there exists a solution $\psi$ of problem (16) satisfying the estimate

$$
\|\psi(t)-\xi(t)\| \leq \varepsilon C_{f, \varphi} E_{\alpha, 1}\left(\beta_{f}\left(\frac{t^{\rho}-a^{\rho}}{\rho}\right)^{\alpha}\right)(\varphi(t)+\eta), \quad t \in[a, b] .
$$

In the following theorem, the Ulam-Hyers-MittagLeffler stability for problem (16) is presented.

Theorem 8. Let $f: J \times \mathbb{R}^{d} \longrightarrow \mathbb{R}^{d}$ be a continuous function which satisfies the assumption as follows. There exists a positive constant $L$ such that, for all $w, z \in \mathbb{R}^{d}$, one has

$$
\|f(t, w)-f(t, z)\| \leq L\|w-z\| .
$$

Then, we have the following assertions:

(A1) For every $\varepsilon>0$, if a function $\xi$ satisfies (64) for all $t \in J$, then there exists a unique solution $\psi$ of problem (16) with initial conditions $\psi(a)=$ $\xi(a), \psi^{\prime}(a)=\xi^{\prime}(a)$, which satisfies

$$
\|\psi(t)-\xi(t)\| \leq \frac{\varepsilon}{L} E_{\alpha, 1}\left(L\left(\frac{t^{\rho}-a^{\rho}}{\rho}\right)^{\alpha}\right), \quad \forall t \in J .
$$

Furthermore, this yields that problem (16) is Ulam-Hyers-Mittag-Leffler stable.

(A2) Assume that the following hypothesis holds: Let $\varphi: J \longrightarrow \mathbb{R}^{+}$in the inequality (65) be a nondecreasing function for all $t \in J$. We assume that there exists a positive constant $K_{\varphi}$ which satisfies $K_{\varphi} L<1$, and for $t \in J$,

$$
\frac{\rho^{1-\alpha}}{\Gamma(\alpha)} \int_{a}^{t} s^{\rho-1}\left(t^{\rho}-s^{\rho}\right)^{\alpha-1} \varphi(s) \mathrm{d} s \leq K_{\varphi} \varphi(t)
$$

Then, for every $\varepsilon>0$, if a function $\xi$ satisfies (65), for all $t \in J$, there exists a unique solution $\psi$ of problem (16) with initial conditions $\psi(a)=\xi(a), \psi^{\prime}(a)=\xi^{\prime}(a)$, and problem (16) is Ulam-Hyers-Rassias-Mittag-Leffler stable.

Proof. In the view of Remark 4 and Theorem 4, we notice that if functions $\xi$ and $\widehat{\xi}$ satisfy inequalities (64) and (65), respectively, then there exists $\delta_{1}(t), \delta_{2}(t) \in C\left(J, \mathbb{R}^{d}\right)$, respectively, such that $\xi(a)=\psi(a), \widehat{\xi}(a)=\psi(a), \xi^{\prime}$ $(a)=\psi^{\prime}(a)$, and $\widehat{\xi}^{\prime}(a)=\psi^{\prime}(a)$, and for $t \in(a, b]$,

$$
\begin{aligned}
& \xi(t)-\xi(a)-\frac{\left(t^{\rho}-a^{\rho}\right)}{\rho} \xi^{(\rho, 1)}(a)=\frac{1}{\Gamma(\alpha)} \int_{a}^{t} s^{\rho-1}\left(\frac{t^{\rho}-s^{\rho}}{\rho}\right)^{\alpha-1}\left[f(s, \xi(s))+\delta_{1}(s)\right] \mathrm{d} s, \\
& \widehat{\xi}(t)-\widehat{\xi}(a)-\frac{\left(t^{\rho}-a^{\rho}\right)}{\rho} \widehat{\xi}^{(\rho, 1)}(a)=\frac{1}{\Gamma(\alpha)} \int_{a}^{t} s^{\rho-1}\left(\frac{t^{\rho}-s^{\rho}}{\rho}\right)^{\alpha-1}\left[f(s, \widehat{\xi}(s))+\delta_{2}(s)\right] \mathrm{d} s,
\end{aligned}
$$

respectively, where $\left\|\delta_{1}(t)\right\| \leq \varepsilon$ and $\left\|\delta_{2}(t)\right\| \leq \varepsilon \varphi(t)$. To show the results of this theorem, the method of successive approximations will be used.
Prove the assertion (A1): we define $\psi_{0}(t)=\xi(t), t \in[a, b]$, and we consider the sequence $\left\{\psi_{n}\right\}_{n \geq 1} \in C\left(J, \mathbb{R}^{d}\right)$ which is defined as follows:

$$
\psi_{n}(t)-\xi(a)-\frac{\left(t^{\rho}-a^{\rho}\right)}{\rho} \xi^{(\rho, 1)}(a)=\frac{\rho^{1-\alpha}}{\Gamma(\alpha)} \int_{a}^{t} s^{\rho-1}\left(\frac{t^{\rho}-s^{\rho}}{\rho}\right)^{\alpha-1} f\left(s, \psi_{n-1}(s)\right) \mathrm{d} s, \quad \forall t \in J
$$


By the abovementioned definition of successive approximations, for $n=1$ and from (71), one has

$$
\begin{aligned}
\left\|\psi_{1}(t)-\psi_{0}(t)\right\|= & \|\left(\psi_{1}(t)-\xi(a)-\frac{\left(t^{\rho}-a^{\rho}\right)}{\rho} \xi^{(\rho, 1)}(a)\right) \\
& -\left(\xi(t)-\xi(a)-\frac{\left(t^{\rho}-a^{\rho}\right)}{\rho} \xi^{(\rho, 1)}(a)\right) \| \\
\leq & \frac{\rho^{1-\alpha}}{\Gamma(\alpha)} \int_{a}^{t} s^{\rho-1}\left(\frac{t^{\rho}-s^{\rho}}{\rho}\right)^{\alpha-1} \delta_{1}(s) \mathrm{d} s \\
\leq & \varepsilon \frac{\left(t^{\rho}-a^{\rho}\right)^{\alpha}}{\rho^{\alpha} \Gamma(\alpha+1)} .
\end{aligned}
$$

By the Lipschitz condition of the function $f$, for any $t \in J$ and $n=2,3,4, \ldots$, one has

$$
\begin{aligned}
\left\|\psi_{n}(t)-\psi_{n-1}(t)\right\|= & \|\left(\psi_{n}(t)-\xi(a)-\frac{\left(t^{\rho}-a^{\rho}\right)}{\rho} \xi^{(\rho, 1)}(a)\right) \\
& -\left(\psi_{n-1}(t)-\xi(a)-\frac{\left(t^{\rho}-a^{\rho}\right)}{\rho} \xi^{(\rho, 1)}(a)\right) \| \\
\leq & \frac{L \rho^{1-\alpha}}{\Gamma(\alpha)} \int_{a}^{t} s^{\rho-1}\left(t^{\rho}-s^{\rho}\right)^{\alpha-1} \\
& \cdot\left\|\psi_{n-1}(s)-\psi_{n-2}(s)\right\| \mathrm{d} s
\end{aligned}
$$

So, from (74) and for $n=2$, one obtains

$$
\left\|\psi_{2}(t)-\psi_{1}(t)\right\| \leq \frac{\varepsilon}{L} \frac{L^{2}\left(t^{\rho}-a^{\rho}\right)^{2 \alpha}}{\rho^{2 \alpha} \Gamma(2 \alpha+1)},
$$

and for $n=3$, we also obtain

$$
\left\|\psi_{3}(t)-\psi_{2}(t)\right\| \leq \frac{\varepsilon}{L} \frac{L^{3}\left(t^{\rho}-a^{\rho}\right)^{3 \alpha}}{\rho^{3 \alpha} \Gamma(3 \alpha+1)} .
$$

By using the mathematical induction method, for $n \geq 4$, we have

$$
\left\|\psi_{n}(t)-\psi_{n-1}(t)\right\| \leq \frac{\varepsilon}{L} \frac{L^{n}\left(t^{\rho}-a^{\rho}\right)^{n \alpha}}{\rho^{n \alpha} \Gamma(n \alpha+1)} .
$$

Now, if we assume that (78) holds for $n=k$, then by (4) one obtains

$$
\begin{aligned}
\left\|\psi_{k+1}(t)-\psi_{k}(t)\right\| \leq & \frac{L}{\Gamma(\alpha)} \int_{a}^{t} s^{\rho-1}\left(\frac{t^{\rho}-s^{\rho}}{\rho}\right)^{\alpha-1} \\
& \cdot\left\|\psi_{k}(s)-\psi_{k-1}(s)\right\| \mathrm{d} s \\
\leq & \frac{L \rho^{1-\alpha}}{\Gamma(\alpha)} \int_{a}^{t} s^{\rho-1}\left(t^{\rho}-s^{\rho}\right)^{\alpha-1} \frac{\varepsilon}{L} \frac{L^{k}\left(s^{\rho}-a^{\rho}\right)^{k \alpha}}{\rho^{k \alpha} \Gamma(k \alpha+1)} \mathrm{d} s \\
\leq & \frac{\varepsilon}{L} \frac{L^{k+1}}{\Gamma((k+1) \alpha+1)}\left(\frac{t^{\rho}-a^{\rho}}{\rho}\right)^{(k+1) \alpha} .
\end{aligned}
$$

which is the inequality (78) for $n=k+1$. This yields that the inequality (78) is satisfied for all $n \geq 1$. Then, one has

$$
\sum_{n=1}^{\infty}\left\|\psi_{n}(t)-\psi_{n-1}(t)\right\| \leq \frac{\varepsilon}{L} \sum_{n=1}^{\infty} \frac{\left[L\left(t^{\rho}-a^{\rho}\right)^{\alpha}\right]^{n}}{\rho^{\alpha n} \Gamma(n \alpha+1)} \leq \frac{\varepsilon}{L}\left[E_{\alpha, 1}\left(L\left(\frac{t^{\rho}-a^{\rho}}{\rho}\right)^{\alpha}\right)-1\right] .
$$

Since the series of the right-hand side of the above inequality is convergent to

$$
\frac{\varepsilon}{L}\left[E_{\alpha, 1}\left(L\left(\frac{t^{\rho}-a^{\rho}}{\rho}\right)^{\alpha}\right)-1\right]
$$

that is,

$$
\sum_{n=1}^{\infty}\left\|\psi_{n}(t)-\psi_{n-1}(t)\right\| \leq \frac{\varepsilon}{L}\left[E_{\alpha, 1}\left(L\left(\frac{t^{\rho}-a^{\rho}}{\rho}\right)^{\alpha}\right)-1\right] .
$$

This yields that the series $\psi_{0}(t)+\sum_{n=1}^{\infty}\left[\psi_{n}(t)-\psi_{n-1}(t)\right]$ is uniformly convergent on $J$ with respect to the norm $\|\cdot\|$. Now, assume that

$$
\bar{\psi}(t)=\psi_{0}(t)+\sum_{n=1}^{\infty}\left[\psi_{n}(t)-\psi_{n-1}(t)\right]
$$

Then, we take $\psi_{k}(t)$ which is the $k^{\text {th }}$ partial of series (83) of the form

$$
\psi_{k}(t)=\psi_{0}(t)+\sum_{n=1}^{k}\left[\psi_{n}(t)-\psi_{n-1}(t)\right], \quad \forall t \in J
$$

By (83) and (84), we notice

$$
\lim _{k \longrightarrow \infty}\left\|\psi_{k}(t)-\bar{\psi}(t)\right\|=0, \quad \forall t \in J
$$

Set $\psi(t)=\bar{\psi}(t)$ for $t \in J$. We prove that the limit function $\psi$ is a solution of the following integral equation on $J:$

$$
\psi(t)-\xi(a)-\frac{\left(t^{\rho}-a^{\rho}\right)}{\rho} \xi^{(\rho, 1)}(a)=\frac{\rho^{1-\alpha}}{\Gamma(\alpha)} \int_{a}^{t} s^{\rho-1}\left(t^{\rho}-s^{\rho}\right)^{\alpha-1} f(s, \psi(s)) \mathrm{d} s .
$$


By using definition of successive approximation for any $t \in J$ and by the Lipschitz condition of $f$, we have

$$
\begin{aligned}
& \left\|\psi(t)-\xi(a)-\frac{\left(t^{\rho}-a^{\rho}\right)}{\rho} \xi^{(\rho, 1)}(a)-\frac{\rho^{1-\alpha}}{\Gamma(\alpha)} \int_{a}^{t} s^{\rho-1}\left(t^{\rho}-s^{\rho}\right)^{\alpha-1} f(s, \psi(s)) \mathrm{d} s\right\| \\
& \leq\left\|\bar{\psi}(t)-\psi_{k}(t)+\frac{\rho^{1-\alpha}}{\Gamma(\alpha)} \int_{a}^{t} s^{\rho-1}\left(t^{\rho}-s^{\rho}\right)^{\alpha-1} f\left(s, \psi_{k-1}(s)\right) \mathrm{d} s-\frac{\rho^{1-\alpha}}{\Gamma(\alpha)} \int_{a}^{t} s^{\rho-1}\left(t^{\rho}-s^{\rho}\right)^{\alpha-1} f(s, \psi(s)) \mathrm{d} s\right\| \\
& \quad \leq\left\|\bar{\psi}(t)-\psi_{k}(t)\right\|+\frac{L \rho^{1-\alpha}}{\Gamma(\alpha)} \int_{a}^{t} s^{\rho-1}\left(t^{\rho}-s^{\rho}\right)^{\alpha-1}\left\|\psi(s)-\psi_{k-1}(s)\right\| \mathrm{d} s .
\end{aligned}
$$

On the other hand, (83) and (84) yield

$$
\begin{aligned}
\left\|\bar{\psi}(t)-\psi_{k}(t)\right\| & =\left\|\sum_{n=k+1}^{\infty}\left[\psi_{n}(t)-\psi_{n-1}(t)\right]\right\| \\
& \leq \sum_{n=k+1}^{\infty}\left\|\psi_{n}(t)-\psi_{n-1}(t)\right\| .
\end{aligned}
$$

Therefore, we obtain for $t \in J$

$$
\left\|\psi(t)-\psi_{k}(t)\right\|=\left\|\bar{\psi}(t)-\psi_{k}(t)\right\| \leq \frac{\varepsilon}{L} \sum_{n=k+1}^{\infty} \frac{\left[L\left(t^{\rho}-a^{\rho}\right)^{\alpha}\right]^{n}}{\rho^{\alpha n} \Gamma(n \alpha+1)} .
$$

So, it follows from (87) that, for $t \in J$,

$$
\begin{aligned}
& \left\|\psi(t)-\xi(a)-\frac{\left(t^{\rho}-a^{\rho}\right)}{\rho} \xi^{(\rho, 1)}(a)-\frac{\rho^{1-\alpha}}{\Gamma(\alpha)} \int_{a}^{t} s^{\rho-1}\left(\frac{t^{\rho}-s^{\rho}}{\rho}\right)^{\alpha-1} f(s, \psi(s)) \mathrm{d} s\right\| \\
& \quad \leq \frac{\varepsilon}{L} \sum_{n=k+1}^{\infty} \frac{\left[L\left(t^{\rho}-a^{\rho}\right)^{\alpha}\right]^{n}}{\rho^{\alpha n} \Gamma(n \alpha+1)}+\frac{\varepsilon}{L} \frac{L}{\Gamma(\alpha)} \int_{a}^{t} s^{\rho-1}\left(t^{\rho}-s^{\rho}\right)^{\alpha-1} \sum_{n=k+1}^{\infty} \frac{\left[L\left(s^{\rho}-a^{\rho}\right)^{\alpha}\right]^{n}}{\rho^{\alpha n} \Gamma(n \alpha+1)} \mathrm{d} s \\
& \quad=\frac{\varepsilon}{L} \sum_{n=k+1}^{\infty} \frac{\left[L\left(t^{\rho}-a^{\rho}\right)^{\alpha}\right]^{n}}{\rho^{\alpha n} \Gamma(n \alpha+1)}+\frac{\varepsilon}{L} \sum_{n=k+1}^{\infty} \frac{\left[L\left(t^{\rho}-a^{\rho}\right)^{\alpha}\right]^{n+1}}{\rho^{\alpha(n+1)} \Gamma((n+1) \alpha+1)} .
\end{aligned}
$$

Then, by taking limit $k \longrightarrow \infty$, the right-hand side of (90) tends to 0 . This yields (86) is a solution of problem (16) with initial conditions $\psi(a)=\xi(a), \psi^{\prime}(a)=\xi^{\prime}(a)$. In addition, from (82) and (83), we also get the following estimation between the solution of problem (16) $\psi(t)$ and the solution of inequality (64):

$$
\|\psi(t)-\xi(t)\| \leq \frac{\varepsilon}{L}\left[E_{\alpha, 1}\left(L\left(\frac{t^{\rho}-a^{\rho}}{\rho}\right)^{\alpha}\right)-1\right] \leq \frac{\varepsilon}{L} E_{\alpha, 1}\left(L\left(\frac{t^{\rho}-a^{\rho}}{\rho}\right)^{\alpha}\right), \quad \forall t \in J
$$

Then, by Definition 1-(i) we can deduce problem (16) is Ulam-Hyers-Mittag-Leffler stable, where $C_{f}:=1 / L$ and $\beta_{f}:=L$.
Prove the assertion (A2): similar to the proof of the assertion (I), we also consider the sequence $\left\{\psi_{n}\right\}_{n \geq 0} \in C\left([a, b], \mathbb{R}^{d}\right)$ given by $\psi_{0}(t)=\widehat{\xi}(t), t \in[a, b]$, and for $n \geq 1$,

$$
\psi_{n}(t)-\widehat{\xi}(a)-\frac{\left(t^{\rho}-a^{\rho}\right)}{\rho} \widehat{\xi}^{(\rho, 1)}(a)=\frac{\rho^{1-\alpha}}{\Gamma(\alpha)} \int_{a}^{t} s^{\rho-1}\left(t^{\rho}-s^{\rho}\right)^{\alpha-1} f\left(s, \psi_{n-1}(s)\right) \mathrm{d} s, \quad \forall t \in[a, b]
$$


By similar processing as the case of (A1), we get for $n \geq 1$ and for $t \in J$,

$$
\left\|\psi_{n}(t)-\psi_{n-1}(t)\right\| \leq \frac{\varepsilon}{L}\left(K_{\varphi} L\right)^{n} \varphi(t) .
$$

In order to check the validity of (93), for $n=1$, from (72) and by using the definition of successive approximations (92), one obtains

$$
\begin{aligned}
\left\|\psi_{1}(t)-\psi_{0}(t)\right\| & =\left\|\left(\psi_{1}(t)-\widehat{\xi}(a)-\frac{\left(t^{\rho}-a^{\rho}\right)}{\rho} \widehat{\xi}^{(\rho, 1)}(a)\right)-\left(\hat{\xi}(t)-\widehat{\xi}(a)-\frac{\left(t^{\rho}-a^{\rho}\right)}{\rho} \widehat{\xi}^{(\rho, 1)}(a)\right)\right\| \\
& \leq \frac{\rho^{1-\alpha}}{\Gamma(\alpha)} \int_{a}^{t} s^{\rho-1}\left(t^{\rho}-s^{\rho}\right)^{\alpha-1}\left\|\delta_{2}(s)\right\| \mathrm{d} s \\
& \leq \varepsilon K_{\varphi} \varphi(t) .
\end{aligned}
$$

Suppose that (93) is true for $n=k \in \mathbb{N}$, that is,

$$
\left\|\psi_{k}(t)-\psi_{k-1}(t)\right\| \leq \frac{\varepsilon}{L}\left(K_{\varphi} L\right)^{k} \varphi(t) .
$$

Then, by the Lipschitz condition of $f$ and the hypothesis of the assertion (A2), we obtain

$$
\begin{aligned}
\left\|\psi_{k+1}(t)-\psi_{k}(t)\right\| & =\left\|\left(\psi_{k+1}(t)-\widehat{\xi}(a)-\frac{\left(t^{\rho}-a^{\rho}\right)}{\rho} \widehat{\xi}^{(\rho, 1)}(a)\right)-\left(\psi_{k}(t)-\widehat{\xi}(a)-\frac{\left(t^{\rho}-a^{\rho}\right)}{\rho} \widehat{\xi}^{(\rho, 1)}(a)\right)\right\| \\
& \leq \frac{L \rho^{1-\alpha}}{\Gamma(\alpha)} \int_{a}^{t} s^{\rho-1}\left(t^{\rho}-s^{\rho}\right)^{\alpha-1}\left\|\psi_{k}(s)-\psi_{k-1}(s)\right\| \mathrm{d} s \\
& \leq \frac{L \rho^{1-\alpha}}{\Gamma(\alpha)} \int_{a}^{t} s^{\rho-1}\left(t^{\rho}-s^{p}\right)^{\alpha-1} \frac{\varepsilon}{L}\left(K_{\varphi} L\right)^{k} \varphi(s) \mathrm{d} s \\
& \leq \frac{\varepsilon}{L}\left(K_{\varphi} L\right)^{k+1} \varphi(t) .
\end{aligned}
$$

This proves inequality (93) is valid for all $n \geq 1$. On the other hand, by the hypothesis $K_{\varphi} L \in(0,1)$ and from (93) we have that, for $t \in J$,

$$
\sum_{n=1}^{\infty}\left\|\psi_{n}(t)-\psi_{n-1}(t)\right\| \leq \varepsilon \varphi(t) \sum_{n=1}^{\infty}\left(K_{\varphi} L\right)^{n} \leq \varepsilon \frac{K_{\varphi}}{1-K_{\varphi} L} \varphi(t) .
$$

Since the function $\varphi(t)$ is continuous on $J$, it is bounded. So, inequality (97) yields that the series $\psi_{0}(t)+\sum_{n=1}^{\infty}\left[\psi_{n}(t)-\right.$ $\left.\psi_{n-1}(t)\right]$ is absolutely and uniformly convergent on $J$ with respect to the distance $\|\cdot\|$. Therefore, we set, for $t \in J$,

$$
\psi(t)=\psi_{0}(t)+\sum_{n=0}^{\infty}\left[\psi_{n+1}(t)-\psi_{n}(t)\right]
$$

Then, similar to the proof of (A1) we also get the estimation between the solution of problem (16) $\psi(t)$ and the solution of inequality (65) $\bar{\xi}(t)$ as follows:

$$
\|\psi(t)-\widehat{\xi}(t)\| \leq \varepsilon \frac{K_{\varphi}}{1-K_{\varphi} L} \varphi(t), \quad t \in[a, b] .
$$

Taking the integral $I_{a^{+}}^{\alpha, \rho}$ on $[a, b]$ of the order $\alpha \in(1,2)$ on both sides of (99) and using hypothesis (H3), one obtains

$$
\begin{aligned}
\frac{p^{1-\alpha}}{\Gamma(\alpha)} \int_{a}^{t} s^{\rho-1}\left(t^{\rho}-s^{\rho}\right)^{\alpha-1}\|\psi(s)-\xi(s)\| \mathrm{d} s & \leq \varepsilon \frac{K_{\varphi}}{1-K_{\varphi} L} \frac{\rho^{1-\alpha}}{\Gamma(\alpha)} \int_{a}^{t} s^{\rho-1}\left(t^{\rho}-s^{\rho}\right)^{\alpha-1} \varphi(s) \mathrm{d} s \\
& \leq \varepsilon \frac{K_{\varphi}^{2}}{1-K_{\varphi} L} \varphi(t) .
\end{aligned}
$$


This yields that

$$
\begin{aligned}
& \|\psi(t)-\xi(t)\|+\frac{\rho^{1-\alpha}}{\Gamma(\alpha)} \int_{a}^{t} s^{\rho-1}\left(t^{\rho}-s^{\rho}\right)^{\alpha-1}\|\psi(s)-\xi(s)\| \mathrm{d} s \\
& \quad \leq \varepsilon \frac{K_{\varphi}+K_{\varphi}^{2}}{1-K_{\varphi} L} \varphi(t) .
\end{aligned}
$$

Therefore, we have

$$
\begin{aligned}
&\|\psi(t)-\xi(t)\| \leq \varepsilon \frac{K_{\varphi}+K_{\varphi}^{2}}{1-K_{\varphi} L} \varphi(t)+\frac{\rho^{1-\alpha}}{\Gamma(\alpha)} \int_{a}^{t} s^{\rho-1}\left(t^{\rho}-s^{\rho}\right)^{\alpha-1} \\
& \cdot\|\psi(s)-\xi(s)\| \mathrm{d} s .
\end{aligned}
$$

Set $p(t)=\|\psi(t)-\xi(t)\|, q(t)=\varepsilon K_{\varphi}+K_{\varphi}^{2} / 1-K_{\varphi} L \varphi(t)$, $r(t)=1 /(\Gamma(\alpha))$. Then, equation (102) becomes

$$
p(t) \leq q(t)+r(t) \rho^{1-\alpha} \int_{a}^{t} s^{\rho-1}\left(t^{\rho}-s^{\rho}\right)^{\alpha-1} p(s) \mathrm{d} s .
$$

By using the Gronwall inequality in 1, (103) yields that

$$
p(t) \leq q(t)+\int_{a}^{t} \sum_{i=1}^{\infty} \frac{(r(t) \Gamma(\alpha))^{i}}{\Gamma(i \alpha)} s^{\rho-1}\left(t^{\rho}-s^{\rho}\right)^{i \alpha-1} q(s) \mathrm{d} s .
$$

Furthermore, since the function $q(t)$ is nondecreasing on $[a, b]$, we obtain

$$
p(t) \leq q(t) E_{\alpha, 1}\left(r(t) \Gamma(\alpha)\left(\frac{t^{\rho}-a^{\rho}}{\rho}\right)^{\alpha}\right) .
$$

Consequently, we obtain

$$
\|\psi(t)-\xi(t)\| \leq \varepsilon \frac{K_{\varphi}+K_{\varphi}^{2}}{1-K_{\varphi} L} \varphi(t) E_{\alpha, 1}\left(\left(\frac{t^{\rho}-a^{\rho}}{\rho}\right)^{\alpha}\right), \quad \forall t \in[a, b] .
$$

Then, by Definition 1-(iii) we can deduce problem (16) is Ulam-Hyers-Rassias-Mittag-Leffler stable, where $C_{f, \varphi}:=\left(K_{\varphi}+K_{\varphi}^{2} / 1-K_{\varphi} L\right)(\varphi(t))$ and $\beta_{f}:=1$.

\section{Conclusion}

In this work, the global existence and uniqueness of the solution to the Caputo-generalized fractional differential equation are investigated. We discussed the stability in various Ulam-Hyers-Mittag-Leffler's types via the successive approximation method.

\section{Data Availability}

No data were used to support this study.

\section{Conflicts of Interest}

The authors declare that they have no conflicts of interest.

\section{Acknowledgments}

The authors would like to thank the Vietnam National Foundation for Science and Technology Development (NAFOSTED), under grant number 107.02-2017.319.

\section{References}

[1] A. Atangana and D. Baleanu, "New fractional derivatives with nonlocal and non-singular kernel: theory and application to heat transfer model," Thermal Science, vol. 20, no. 2, pp. 763-769, 2016.

[2] D. Baleanu, G. C. Wu, and S. D. Zeng, "Chaos analysis and asymptotic stability of generalized Caputo fractional differential equations," Chaos, Solitons \& Fractals, vol. 102, pp. 1-7, 2017.

[3] E. Demirci and N. Ozalp, "A method for solving differential equations of fractional order," Journal of Computational and Applied Mathematics, vol. 236, no. 11, pp. 2754-2762, 2012.

[4] K. Diethelm, "The analysis of fractional differential equations an application-oriented exposition using differential operators of caputo type," Lecture Notes in Mathematics, SringerVerlag Berlin, Berlin, Germany, 2004.

[5] A. A. Kilbas, H. M. Srivastava, and J. J. Trujillo, "Theory and applications of fractional differential equations," North-Holland Mathematics Studies, Vol. 204, Elsevier, Amsterdam, Netherlands, 2006.

[6] I. Podlubny, Fractional Differential Equations, Academic Press, San Diego, CA, USA, 1999.

[7] U. N. Katugampola, "New approach to a generalized fractional integral," Applied Mathematics and Computation, vol. 218, no. 3, pp. 860-865, 2011.

[8] U. N. Katugampola, "A new approach to generalized fractional derivatives," Bulletin of Mathematical Analysis and Applications, vol. 6, no. 4, pp. 1-15, 2014.

[9] A. Fernandez, M. A. Özarslan, and D. Baleanu, "On fractional calculus with general analytic kernels," Applied Mathematics and Computation, vol. 354, pp. 248-265, 2019.

[10] R. Almeida, "A Gronwall inequality for a general Caputo fractional operator," Mathematical Inequalities \& Applications, vol. 20, no. 4, pp. 1089-1105, 2017.

[11] R. Almeida, A. B. Malinowska, and T. Odzijewicz, "Fractional differential equations with dependence on the CaputoKatugampola derivative," Journal of Computational and Nonlinear Dynamics, vol. 11, p. 11, 2016.

[12] D. R. Anderson and D. J. Ulness, "Properties of the Katugampola fractional deriva- tive with potential application in quantum mechanics," Journal of Mathematical Physics, vol. 56, no. 6, Article ID 063502, 2015.

[13] U. N. Katugampola, "Existence and uniqueness for a class of generalized fractional differential equations," 2016, https:// arxiv.org/abs/1411.5229.

[14] B. Lupinska, T. Odzijewicz, and E. Schmeidel, "On the solutions to a generalized fractional Cauchy problem," Applicable Analysis and Discrete Mathematics, vol. 10, pp. 332-344, 2016.

[15] S. Zeng, D. Baleanu, Y. Bai, and G. Wu, "Fractional differential equations of Caputo-Katugampola type and numerical solutions," Applied Mathematics and Computation, vol. 315, pp. 549-554, 2017.

[16] W. Zhen, H. Xia, L. Ning, and S. Xiao-Na, "Image encryption based on a delayed fractional-order chaotic logistic system," Chinese Physics B, vol. 21, Article ID 050506, , 2012. 
[17] J. Wang and X. $\mathrm{Li}$, "E $\alpha$-Ulam type stability of fractional order ordinary differential equations," Journal of Applied Mathematics and Computing, vol. 45, no. 1-2, pp. 449-459, 2014.

[18] S. Abbas, M. Benchohra, J.-E. Lazreg, and Y. Zhou, "A survey on Hadamard and Hilfer fractional differential equations: analysis and stability," Chaos, Solitons \& Fractals, vol. 102, pp. 47-71, 2017.

[19] N. Eghbali, V. Kalvandi, and J. M. Rassias, "A fixed point approach to the Mittag-Leffler-Hyers-Ulam stability of a fractional integral equation," Open Mathematics, vol. 14, pp. 237-246, 2016.

[20] J. Huang and Y. Li, "Hyers-Ulam stability of delay differential equations of first order," Mathematische Nachrichten, vol. 289, no. 1, pp. 60-66, 2016.

[21] D. H. Hyers, "On the stability of the linear functional equation," Proceedings of the National Academy of Sciences, vol. 27, no. 4, pp. 222-224, 1941.

[22] K. D. Kucche and S. T. Sutar, "Stability via successive approximation for nonlinear implicit fractional differential equations," Moroccan Journal of Pure and Applied Analysis, vol. 3, no. 1, pp. 36-54, 2017.

[23] K. D. Kucche and S. T. Sutar, "On existence and stability results for nonlinear fractional delay differential equations," Boletim da Sociedade Paranaense de Matemática, vol. 36, no. 4, pp. 55-75, 2018.

[24] T. M. Rassias, "On the stability of the linear mapping in Banach spaces," Proceedings of the American Mathematical Society, vol. 72, no. 2, p. 297, 1978.

[25] I. A. Rus, "Ulam stabilities of ordinary differential equations in a Banach space," Carpathian Journal of Mathematics, vol. 26, Article ID 103107, , 2010.

[26] J. V. D. C. Sousa and E. C. D. Oliveira, "UlamHyers stability of a nonlinear fractional Volterra integro-differential equation," Applied Mathematics Letters, vol. 81, pp. 50-56, 2018.

[27] R. Shah and Z. Akbar, "A fixed point approach to the stability of a nonlinear volterra integrodiff erential equation with delay," Hacettepe Journal of Mathematics and Statistics, vol. 47, pp. 615-623, 2018.

[28] E. C. D. Oliveira and J. V. D. C. Sousa, "UlamHyersRassias stability for a class of fractional integro-differential equations," Results in Mathematics, vol. 73, p. 111, 2018.

[29] S. M. Ulam, A Collection of Mathematical Problems, Interscience Publishers, New York, NY, USA, 1968.

[30] J. Wang and Y. Zhang, "Ulam-Hyers-Mittag-Leffler stability of fractional-order delay differential equations," Optimization, vol. 63, no. 8, pp. 1181-1190, 2014.

[31] K. Diethelm and N. J. Ford, "Analysis of fractional differential equations," Journal of Mathematical Analysis and Applications, vol. 265, no. 2, pp. 229-248, 2002. 